\title{
Efeitos de Vizinhança Ortográfica no Português Brasileiro: Um Estudo com a Tarefa de Identificação Perceptual
}

\author{
Orthographic Neighborhood Effects in Brazilian Portuguese: \\ A Study on Perceptual Identification Tasks
}

\author{
Francis Ricardo dos Reis Justi ${ }^{*}, a$ \& Antonio Roazzi ${ }^{b}$ \\ ${ }^{a}$ Universidade Federal de Alagoas, Maceió, Brasil \& ${ }^{b}$ Universidade Federal de Pernambuco, Recife, Brasil
}

\begin{abstract}
Resumo
Esse estudo investigou os efeitos do número de vizinhos ortográficos $(\mathrm{N})$ e do número de vizinhos ortográficos de maior frequência de ocorrência (NF) na tarefa de identificação perceptual. Trinta e dois estudantes de psicologia falantes do português brasileiro participaram do estudo. Os estímulos experimentais consistiram em 64 palavras que atenderam a uma manipulação fatorial 2 × 2 de N e NF. Os participantes apresentaram uma maior porcentagem de erros nas palavras com vizinhos ortográficos de maior frequência e uma menor porcentagem de erros nas palavras com muitos vizinhos ortográficos. Argumentou-se que esses resultados refletem o efeito dessas variáveis no processo de acesso lexical e que os modelos de Dupla Rota e PPD têm dificuldades em acomodá-los.

Palavras-chave: Vizinhança ortográfica, tarefa de identificação perceptual, reconhecimento visual de palavras, leitura.
\end{abstract}

\begin{abstract}
This study investigated the effects of Neighborhood Density (ND) and Neighborhood Frequency (NF) on visual word recognition in 32 Brazilian Portuguese speakers. Participants were given a standard perceptual identification task in which they had a 3-second time-limit for saying aloud what the target word was. Experimental stimuli consisted of 64 words according to an ND (2) x NF (2) factorial design. Higher percentages of errors were made for words with a higher frequency neighbor and lower percentages of errors were made for words with a high neighborhood density. It is argued that the results were due to ND and NF effects on lexical access and that they pose difficulties for Dual Route and PDP models of visual word recognition.

Keywords: Orthographic neighborhood, perceptual identification task, visual word recognition, reading.
\end{abstract}

É a palavra 'carta' similar do ponto de vista ortográfico à palavra 'carga'? Nas línguas alfabéticas as palavras são escritas com base em um pequeno conjunto de símbolos, no caso do português do Brasil, por exemplo, essas são escritas com base em apenas 26 letras. Assim sendo, torna-se inevitável que muitas das palavras escritas em línguas alfabéticas compartilhem um mesmo conjunto de letras e sejam, em certa medida, similares do ponto de

\footnotetext{
* Esse estudo é parte integrante da tese de doutorado do primeiro autor. Assim sendo, os participantes desse estudo participaram também de outros experimentos, porém, esses foram desenvolvidos com estímulos e tarefas diferentes e, por questões de espaço, serão apresentados em outros trabalhos.

Ambos os autores agradecem ao Conselho Nacional de Desenvolvimento Científico e Tecnológico (CNPq) por financiar suas atividades.

Endereço para correspondência: Instituto de Ciências Humanas Comunicação e Artes, Curso de Psicologia, Universidade Federal de Alagoas, Campus A. C. Simões, Av. Lourival Melo Mota, s/n, Bairro Tabuleiro do Martins, Maceió, AL, Brasil 57072-970. E-mail: francisjusti@gmail.com
}

vista ortográfico. Na tradição de pesquisas sobre o reconhecimento visual de palavras existem, pelo menos, duas medidas do grau de similaridade ortográfica de uma palavra. A mais tradicional é a medida N (de Neighbourhood Density) que indexa o número de vizinhos ortográficos da palavra alvo, ou seja, se refere ao número de palavras que são formadas ao se mudar uma letra da palavra alvo enquanto a posição das demais letras é mantida (Coltheart, Davelaar, Jonasson, \& Besner, 1977). Com base nessa medida, a palavra 'carta' teria dez vizinhos ortográficos ('farta', 'parta', 'certa', 'corta', 'curta', 'canta', 'capta', 'casta', 'carga' e 'carma'), enquanto a palavra 'flor' não teria nenhum vizinho ortográfico. Além da medida $\mathrm{N}$, outra medida também bastante utilizada é a medida NF (de Neighbourhood Frequency) que se refere à frequência de ocorrência dos vizinhos da palavra alvo, ou seja, se ela possui vizinhos de frequência de ocorrência maior do que a dela ou não (Grainger, O'Regan, Jacobs, \& Segui, 1989). Por exemplo, no caso de NF, pode-se dizer que a palavra 'alarde' tem um vizinho ortográfico de maior frequência de ocorrência que é a palavra 'alarme', já a 
palavra 'ação' não tem um vizinho ortográfico mais frequente (Justi \& Justi, 2008).

É interessante observar que em uma análise de corpus linguístico realizada por Justi e Justi (2008) constatouse que das palavras do português brasileiro de 4 a 6 letras, aproximadamente, $79 \%$ têm, pelo menos, um vizinho ortográfico e $54 \%$ têm, pelo menos, 1 vizinho ortográfico mais frequente, o que corrobora a hipótese de que em línguas alfabéticas as palavras tendem a ter um alto grau de similaridade ortográfica. Nesse caso, ao se considerar a leitura hábil, pelo menos em sistemas alfabéticos, uma das principais tarefas que o nosso sistema cognitivo tem que desempenhar é a de selecionar a entrada lexical correta diante das diversas palavras que podem ser similares em sua forma ortográfica à palavra alvo. Seria uma palavra ortograficamente similar a muitas outras, reconhecida mais lentamente do que uma palavra pouco similar? O efeito que o grau de similaridade ortográfica de uma palavra exerce sobre o seu reconhecimento é conhecido na literatura como efeito de vizinhança ortográfica (Andrews, 1997; Mathey, 2001; Perea \& Rosa, 2000) e a operacionalização das medidas $\mathrm{N}$ e NF tem o intuito de permitir aos pesquisadores estabelecer se esse efeito seria facilitador ou inibidor. Um efeito facilitador implica que palavras com muitos vizinhos ortográficos e/ou com vizinhos mais frequentes serão reconhecidas com mais precisão e rapidez do que palavras com poucos vizinhos ortográficos e/ou sem vizinhos mais frequentes. Já um efeito inibidor implica o padrão oposto.

As pesquisas sobre o efeito de vizinhança ortográfica têm se revestido de grande importância teórica porque modelos influentes de reconhecimento visual de palavras (Coltheart, Rastle, Perry, Langdon, \& Ziegler, 2001; Plaut, McClelland, Seidenberg, \& Patterson, 1996; Seidenberg \& McClelland, 1989) fazem predições diferentes quanto aos efeitos das variáveis $\mathrm{N}$ e $\mathrm{NF}$ e, dessa forma, essas pesquisas podem ajudar a determinar qual dos modelos é o mais adequado aos dados. Diversos estudos sobre o efeito de vizinhança ortográfica têm sido realizados visando estabelecer a direção desse efeito, porém os resultados ainda não são conclusivos, parecendo variar, em certa medida, de acordo com o tipo de tarefa e língua em que os estudos foram realizados (p.ex.: Andrews, 1989; Arduino \& Burani, 2004; Carreiras, Perea, \& Grainger, 1997; Grainger \& Jacobs, 1996; Grainger et al., 1989; Huntsman \& Lima, 1996, 2002; Justi \& Pinheiro, 2006, 2008; Mathey \& Zagar, 2006; Perea, Carreiras, \& Grainger, 2004; Perea \& Pollatsek, 1998; Pollatsek, Perea, \& Binder, 1999; Sears, Campbell, \& Lupker, 2006; Siakaluk, Sears, \& Lupker, 2002).

O presente estudo procurou contribuir para a clarificação desse debate investigando o efeito de vizinhança ortográfica no português brasileiro por meio de uma tarefa de identificação perceptual. De acordo com Grainger e Jacobs (1996) esse tipo de tarefa seria mais adequado para se estudar o acesso lexical ${ }^{1}$ já que para responder corretamente o participante deve identificar efetivamente qual foi o estímulo apresentado. Destarte, apresenta-se a seguir uma discussão dos estudos realizados com a tarefa de identificação perceptual e o rationale do presente estudo (uma revisão mais extensa da literatura pode ser encontrada nos trabalhos de Andrews, 1997; Mathey, 2001; e, Perea \& Rosa, 2000).

Grainger e Segui (1990) foram os primeiros a investigar o efeito de NF no reconhecimento visual de palavras por meio da tarefa de identificação perceptual. Os pesquisadores se basearam no paradigma do desmascaramento progressivo (progressive demasking task) e realizaram sua pesquisa com estudantes universitários falantes do Francês. No estudo de Grainger e Segui (1990), a palavra alvo aparecia por completo (p. ex.: 'bille'), na tela de um computador, e rapidamente era substituída, ciclicamente, por uma máscara (p. ex.: '\#\#\#\#'). A cada ciclo, palavra/máscara, a exposição da palavra alvo era aumentada em 16 milissegundos (ms) e a da máscara diminuída na mesma quantidade. Os participantes tinham que pressionar uma tecla assim que identificassem a palavra alvo. Após isso, a palavra alvo desaparecia e os participantes deviam digitar qual era essa palavra. As palavras utilizadas como estímulo pelos experimentadores atenderam a uma manipulação fatorial de NF (nenhum vizinho de maior frequência e, pelo menos, um vizinho de maior frequência) e da frequência de ocorrência das palavras (palavras de baixa frequência de ocorrência e palavras de média frequência de ocorrência). Foram controlados a frequência de ocorrência dos bigramas e o número de vizinhos ortográficos $(\mathrm{N})$ das palavras. Encontrou-se um efeito inibidor de NF e um efeito facilitador da frequência de ocorrência das palavras no tempo de reação. Isso é, as palavras que tinham pelo menos um vizinho ortográfico de maior frequência foram reconhecidas mais lentamente do que as que não tinham, e, as palavras de média frequência de ocorrência foram reconhecidas mais rapidamente do que as de baixa frequência. $\mathrm{Na}$ análise da porcentagem de erros apenas o efeito inibidor de NF foi estatisticamente significativo.

Em outro estudo, Grainger e Jacobs (1996) tentaram replicar os achados de Grainger e Segui (1990) na tarefa 
de identificação perceptual, utilizando dois paradigmas: o do desmascaramento progressivo e a versão padrão da tarefa de identificação perceptual (standard perceptual identification task). O estudo utilizando o paradigma do desmascaramento progressivo contou com a participação de trinta alunos de um curso de Psicologia, todos falantes da língua francesa. Os autores utilizaram os mesmos procedimentos de Grainger e Segui (1990), porém dessa vez, utilizaram apenas palavras de baixa frequência de ocorrência e observaram um efeito inibidor de NF e um efeito nulo de N. O estudo com a tarefa de identificação perceptual padrão também foi realizado com trinta alunos de um curso de Psicologia, todos falantes da língua francesa e que não tinham participado do experimento anterior. Esse novo estudo teve como base o seguinte paradigma: aparecia no centro da tela uma máscara (\#\#\#\#\#) por $500 \mathrm{~ms}$, sendo imediatamente seguida pela palavra alvo que permanecia na tela por $40 \mathrm{~ms}$ que, por fim, era substituída novamente por uma máscara (\#\#\#\#) que permanecia na tela até que o participante digitasse a palavra que tinha visto. As palavras utilizadas como estímulo pelos experimentadores atenderam a uma manipulação fatorial de NF (nenhum vizinho de maior frequência e, pelo menos, um vizinho de maior frequência) e da frequência de ocorrência das palavras (palavras de baixa frequência de ocorrência e palavras de alta frequência de ocorrência), sendo que o número de vizinhos ortográficos $(\mathrm{N})$ foi mantido constante (média de 2,5 vizinhos ortográficos). Os resultados evidenciaram que as palavras de maior frequência de ocorrência foram reconhecidas de forma mais precisa, enquanto o efeito inibidor de NF foi significativo apenas para as palavras de baixa frequência de ocorrência. Levando-se em consideração que no estudo anterior de Grainger e Segui (1990) não foram utilizadas palavras de alta frequência de ocorrência, os resultados desses estudos tornam-se bastante consistentes.

Em um estudo com universitários falantes do espanhol, Carreiras et al. (1997) utilizaram a mesma metodologia empregada por Grainger e Segui (1990) em sua tarefa de identificação perceptual. As palavras-estímulo empregadas por Carreiras et al. (1997) eram palavras de baixa frequência de ocorrência e atendiam a uma manipulação fatorial de $\mathrm{N}$ (palavras com poucos vizinhos ortográficos e palavras com muitos vizinhos ortográficos) e NF (palavras sem vizinhos ortográficos de maior frequência e palavras com, pelo menos, um vizinho ortográfico de maior frequência), enquanto a frequência das sílabas, o som inicial e o número de letras das palavras foram controlados. Os pesquisadores encontraram um efeito inibidor estatisticamente significante de NF e um efeito nulo de N.

Em um estudo utilizando outra variação da tarefa de identificação perceptual, Snodgrass e Mintzer (1993) desenvolveram cinco experimentos para investigar o efeito de $\mathrm{N}$ no reconhecimento visual de palavras em estudantes universitários falantes da língua inglesa. Na tarefa de identificação perceptual empregada por Snodgrass e
Mintzer, fragmentos da palavra alvo foram apresentados de maneira sucessiva e incremental, no centro da tela de um computador. Os participantes controlavam o aparecimento dos fragmentos da palavra, que iam sempre da versão mais fragmentada da palavra alvo (mais difícil de reconhecer) à menos fragmentada (eram oito níveis de fragmentação, sendo que o último nível correspondia à palavra não fragmentada). Os participantes eram orientados a digitar qual era a palavra alvo o quanto antes. Em seus dois primeiros experimentos, Snodgrass e Mintzer (1993) permitiram aos participantes que adotassem tentativas sucessivas de acertar a palavra alvo e obtiveram resultados inconsistentes: um efeito nulo de N, no primeiro experimento e, um efeito facilitador, no segundo. No terceiro e quarto experimentos os autores permitiram aos participantes apenas uma tentativa de acertar o estímulo e, nesse caso, observaram um efeito inibidor de $\mathrm{N}$ que se manteve consistente em ambos os experimentos. Por fim, Snodgrass e Mintzer desenvolveram um último experimento. Nesse experimento, os participantes não controlavam o aparecimento dos diferentes níveis de fragmentação. Os oito níveis de fragmentação apareciam em sucessão de $167 \mathrm{~ms}$ cada e os participantes deviam pressionar uma tecla assim que tivessem identificado a palavra alvo, quando então eram solicitados a digitá-la (apenas uma tentativa de identificar a palavra era permitida). Consistente com os resultados do terceiro e quarto experimentos, os pesquisadores encontraram um efeito inibidor de $\mathrm{N}$ em seu quinto experimento.

Para Andrews (1997) um dos problemas dessa modalidade das tarefas de identificação perceptual é que os participantes podem utilizar diversas estratégias para tentar adivinhar a palavra correta à medida que as letras vão aparecendo e, talvez, isso não implique em acesso lexical genuíno. Para Andrews, a variação nos resultados obtidos, de acordo com o número de tentativas permitidas, seria um indício do uso dessas estratégias. Essa crítica é particularmente pertinente para os experimentos nos quais os participantes podiam controlar o aparecimento dos níveis de fragmentação das palavras, pois poderiam fazer hipóteses a respeito do estímulo antes de passar para o próximo nível de fragmentação. No entanto, o quinto experimento de Snodgrass e Mintzer (1993) é menos suscetível a essa crítica, porque os participantes não controlavam o aparecimento dos diferentes níveis de fragmentação. Nesse caso, a dinâmica temporal de aparecimento do estímulo alvo no quinto experimento do estudo de Snodgrass e Mintzer, pode ser considerada semelhante a de outros estudos. Além disso, nos estudos de Carreiras et al. (1997) e de Grainger e Segui (1990), a palavra alvo aparecia por completo e era coberta, ciclicamente, por uma máscara, assim o processo perceptual não poderia ser afetado pela natureza incremental da tarefa como proposto por Andrews (1997)

Levando em conta essas críticas de Andrews (1997) e visando clarificar o papel de N e NF na tarefa de identificação perceptual Sears, Lupker e Hino (1999) desenvolve- 
ram uma série de quatro estudos com a versão padrão dessa tarefa. Sears, Lupker et al. (1999) dividiram a tarefa de identificação perceptual em duas versões: uma em que a visibilidade dos estímulos era maior e outra em que a visibilidade dos estímulos era menor. Em ambas as versões a palavra alvo era apresentada por apenas $24 \mathrm{~ms}$, sendo a principal diferença entre as versões o tempo de duração das máscaras (\#\#\#\#): na versão de maior visibilidade, as máscaras (prévia ao estímulo e posterior a esse) eram apresentadas por $500 \mathrm{~ms}$; enquanto na versão de menor visibilidade, as máscaras eram apresentadas por apenas $42 \mathrm{~ms}$. A resposta consistia em digitar a palavra alvo.

Nos seus dois primeiros experimentos, Sears, Lupker et al. (1999) manipularam a frequência de ocorrência das palavras (palavras de baixa e alta frequência de ocorrência), o número de vizinhos ortográficos (palavras com poucos vizinhos ortográficos e palavras com muitos vizinhos ortográficos) e NF (palavras sem vizinhos ortográficos de maior frequência de ocorrência e palavras com pelo menos um vizinho ortográfico de maior frequência de ocorrência). Participaram dos experimentos 82 estudantes universitários falantes da língua inglesa, sendo que 36 participaram da versão na qual os estímulos tinham maior visibilidade e 46 da versão em que os estímulos tinham menor visibilidade. As palavras de maior frequência de ocorrência foram reconhecidas mais corretamente em ambas as versões dos experimentos. Já o efeito de vizinhança ortográfica apresentou um padrão diferente de acordo com a versão. Na versão onde a visibilidade dos estímulos era maior, $\mathrm{N}$ apresentou um efeito inibidor geral e NF apresentou um efeito inibidor que se restringiu às palavras de baixa frequência de ocorrência que tinham poucos vizinhos ortográficos. Já na condição em que a visibilidade dos estímulos era menor, tanto $\mathrm{N}$ quanto $\mathrm{NF}$ apresentaram um efeito facilitador. Na tentativa de compreender melhor o efeito de NF, Sears, Lupker et al. (1999) desenvolveram mais dois experimentos com falantes da língua inglesa, porém, dessa vez, manipularam apenas NF, sendo que todas as palavras alvo tinham muitos vizinhos ortográficos e variavam em frequência de ocorrência (alta e baixa). Novamente, duas condições de visibilidade foram empregadas. $\mathrm{Na}$ condição de maior visibilidade o efeito de NF foi nulo e na condição de menor visibilidade o efeito de NF foi facilitador. Na interpretação de seus dados, Sears, Lupker et al. (1999) argumentaram que, na condição de maior visibilidade, os participantes teriam maior probabilidade de tentar adivinhar a palavra alvo e isso levaria a um efeito inibidor, porque quanto maior o número de vizinhos ortográficos de uma palavra, maior a probabilidade dos participantes "chutarem" um desses vizinhos ao invés da palavra alvo. Já na condição de menor visibilidade, os participantes "chutariam" menos, com isso, o processo de acesso lexical seria menos "contaminado" por estratégias sofisticadas de adivinhação e o efeito observado de N e NF deveria ser facilitador. O problema dessa interpretação de Sears, Lupker et al. (1999) é que ela tem como base a premissa um tanto contra-intuitiva de que os participantes vão tender a "chutar" mais vezes nas condições fáceis (condições de maior visibilidade) do que nas condições mais difíceis (condições de menor visibilidade). Ora, parece fazer mais sentido que as pessoas tentem "chutar" uma resposta nas situações em que elas têm menos clareza do que viram do que nas situações nas quais elas têm mais clareza do que podem ter visto.

Independente de qual interpretação possa fazer mais sentido, é importante se considerar que ambas são possíveis e que, talvez, o principal seja tentar reduzir ao máximo a possibilidade de utilização de estratégias de adivinhação por parte dos participantes de forma a tentar garantir que o acesso lexical na tarefa em questão não esteja sendo "contaminado" por essas. Na maioria dos estudos realizados com a tarefa de identificação perceptual (Grainger \& Jacobs, 1996; Grainger \& Segui, 1990; Sears, Lupker et al., 1999; Snodgrass \& Mintzer, 1993) os participantes não tinham um limite de tempo para responder (digitar a palavra alvo) e isso pode encorajar o uso de estratégias para tentar adivinhar qual é a palavra mais compatível com o estímulo visto, independentemente da condição de visibilidade dos estímulos. Além disso, como nesses estudos a resposta consistia em digitar qual era a palavra que tinha sido apresentada, existe a possibilidade de que processos específicos relacionados à grafia das palavras possam afetar de alguma forma a resposta dos participantes (Goldrick, Folk, \& Rapp, 2004), o que é indesejável, já que o interesse dos pesquisadores é o efeito de vizinhança ortográfica na leitura.

Considerando essas dificuldades dos trabalhos anteriores, o presente estudo teve como objetivo investigar os efeitos de N e NF na versão padrão da tarefa de identificação perceptual. Para evitar as críticas enfrentadas pelos trabalhos anteriores, o presente estudo fez uso de uma tarefa de identificação perceptual, na qual foram tomados os seguintes cuidados: (a) estabeleceu-se um limite de tempo (3 segundos) para a resposta dos participantes e (b) a resposta requisitada consistia em se dizer em voz alta o nome da palavra que tinha sido apresentada. Dessa forma, pode-se esperar que qualquer tentativa sofisticada de adivinhação dos estímulos tenha sido reduzida, porque os participantes tiveram um limite curto de tempo para responder. Além disso, ao requisitar respostas vocais, esse estudo procurou evitar que processos específicos relacionados ao ato de digitar as palavras pudessem afetar os resultados. Por fim, se optou por utilizar a versão padrão da tarefa de identificação perceptual nesse trabalho porque apenas dois estudos utilizaram essa tarefa e apresentaram resultados relativamente conflitantes (Grainger \& Jacobs, 1996; Sears, Lupker et al., 1999). Assim sendo, é importante investigar se os efeitos de $\mathrm{Ne}$ NF são generalizáveis para essa tarefa também. 
Justi, F. R. R. \& Roazzi, A. (2012). Efeitos de Vizinhança Ortográfica no Português Brasileiro: Um Estudo com a Tarefa de Identificação Perceptual.

\section{Método}

\section{Participantes}

Participaram desse experimento 32 alunos do curso de Psicologia da Universidade Federal de Alagoas (UFAL), todos falantes do português brasileiro. A média de idade dos participantes foi de 21 anos e 2 meses, sendo 24 do sexo feminino e 8 do sexo masculino. Esse estudo foi aprovado pelo Comitê de Ética em Pesquisa da UFAL (número de registro 018238/2008-06).

\section{Material}

Os estímulos experimentais consistiram de 64 palavras que atenderam a uma manipulação fatorial $2 \times 2$ de $\mathrm{N}$ (um vizinho ortográfico ou quatro ou mais vizinhos ortográficos) e NF (sem vizinhos ortográficos de maior frequência de ocorrência ou com um vizinho ortográfico de maior frequência). Essas 64 palavras foram retiradas do trabalho de Justi e Justi (2008) que traz estatísticas de vizinhança ortográfica para uma amostra de 8465 palavras do Corpus NILC / São Carlos (Núcleo Interinstitucional de Lingüística Computacional [NILC], 2005). Além dessas 64 palavras atenderem a uma manipulação fatorial de N e NF, as seguintes características dessas também foram controladas: a frequência de ocorrência (foram utilizadas palavras de baixa frequência de ocorrência, tendo essas, em média, 10,11 ocorrências por milhão de palavras); o número de sílabas (todas dissílabas); o grau de correspondência no mapeamento grafema-fonema (todas regulares); a classe gramatical (apenas substantivos); e a natureza do fonema inicial (as palavras foram pareadas entre as condições experimentais de acordo com seu fonema inicial). Por fim, além dessas palavras, esse experimento contou também com outras 12 palavras que foram utilizadas na sessão de treinamento. Esses estímulos tinham características semelhantes às das palavras utilizadas na sessão experimental.

\section{Procedimentos}

As 64 palavras correspondentes à manipulação fatorial de N e NF foram subdivididas em quatro listas (pareadas pela natureza do fonema inicial e balanceadas pela frequência de ocorrência média) que foram cruzadas entre as quatro condições experimentais. A ordem de apresentação dessas listas foi contrabalanceada entre as quatro condições experimentais, de forma que cada participante foi exposto a apenas uma lista por condição experimental, configurando, assim, um total de 16 palavras ${ }^{2}$. Assim sendo, na sessão experimental, cada participante foi exposto a 16 palavras e, na sessão de treinamento, cada um foi exposto a 12 palavras. A lista com as 64 palavras que compuseram a sessão experimental desse estudo encontra-se no Anexo A.

Os participantes foram testados individualmente em uma sala cedida pela UFAL, onde foram recebidos e instruídos por um colaborador do pesquisador quanto aos procedimentos. É importante acrescentar que o colaborador não tinha conhecimento das hipóteses experimentais, sendo esse um experimento do tipo duplo-cego. $\mathrm{O}$ instrumento utilizado para o teste foi um computador de arquitetura compatível à IBM-PC e o software utilizado para a apresentação dos estímulos e coleta dos dados de tempo de reação $(\mathrm{TR})^{3}$ e porcentagem de erros foi o DMDX (Forster \& Forster, 2003) ( $^{4}$ O participante se acomodava na cadeira, ficando a uma distância aproximada de 40 centímetros da tela do computador, dependendo de sua postura. Após se acomodar, o colaborador solicitava ao participante que falasse algumas palavras com a finalidade de calibrar o microfone e que lesse as instruções experimentais na tela do computador. As instruções foram as seguintes:

Nesse teste, é necessário que você preste muita atenção. Esse símbolo + indicará onde você deve olhar. Logo depois, esse símbolo \#\#\#\#\# aparecerá brevemente na tela. Em seguida, uma palavra aparecerá muito rapidamente, sendo coberta pelo símbolo \#\#\#\#\# e depois por ??????. Você deve tentar dizer qual era a palavra o mais rápido que puder. Aperte o ENTER para iniciar o treinamento.

Após ler essas instruções o participante tinha a oportunidade de redimir quaisquer dúvidas antes de iniciar a sessão de treinamento ou logo após essa, porém, antes de iniciar a sessão experimental.

Os estímulos foram apresentados em uma configuração de tela de 640x480 pixels, na fonte 'fixedsys' de tamanho 10 e em letras capitulares, sendo a cor da fonte branca e o fundo azul. A exposição dos estímulos teve a seguinte dinâmica de apresentação na sessão experimental: uma marca de fixação $(+)$ aparecia na tela por $500 \mathrm{~ms}$ e depois desaparecia deixando a tela sem estímulos por 500ms; aparecia então uma máscara (\#\#\#\#\#) que durava $500 \mathrm{~ms}$, sendo apagada e imediatamente seguida pela
${ }^{2} \mathrm{O}$ esquema de contrabalanceamento utilizado foi o mesmo proposto por Pollatsek e Well (1995) no anexo 'B' de seu trabalho. Esse contrabalanceamento de listas permite o cálculo da variação referente ao efeito principal das listas e da variação referente à interação entre listas e tratamento. Isso permitiu que na análise estatística realizada nesse estudo a variação referente à interação entre listas e tratamento fosse acrescentada ao termo de erro do teste estatístico, evitando dessa forma o problema conhecido como a "falácia da linguagem como um efeito fixo" (Raaijmakers, Schrijnemakers, \& Gremmen, 1999). Veja-se o anexo B do trabalho de Pollatsek e Well (1995) para uma discussão detalhada do design utilizado nesse estudo. Os trabalhos de Clark (1973), Raaijmakers (2003) e de Raaijmakers et al. (1999) discutem o problema da "falácia da linguagem como um efeito fixo".

${ }^{3}$ Mensurado pelo tempo decorrido entre o aparecimento do estímulo e o início da resposta vocal emitida pelo participante.

${ }^{4}$ O software DMDX (Forster \& Forster, 2003) sincroniza a apresentação dos estímulos com o refresh rate do monitor o que permite uma precisão de milissegundos na dinâmica de apresentação dos estímulos e no registro do tempo de reação dos participantes. Além disso, o software também gera uma ordem aleatória de apresentação dos estímulos para cada participante. 
apresentação da palavra alvo que durava $60 \mathrm{~ms}$; então a palavra alvo desaparecia sendo imediatamente substituída por uma máscara (\#\#\#\#\#) que durava $500 \mathrm{~ms}$; por fim, a máscara era substituída por sinais de interrogação (??????) que duravam até o tempo limite para uma resposta (3 segundos contados a partir da apresentação da palavra alvo). O participante devia dizer em voz alta qual era a palavra apresentada em até 3 segundos. A sessão de treinamento seguiu a mesma dinâmica, porém contou com feedback quanto à resposta dada. Além disso, o tempo de apresentação da palavra alvo na sessão de treinamento iniciou em $100 \mathrm{~ms}$, decrescendo em $20 \mathrm{~ms}$ a cada duas tentativas até atingir o piso de $60 \mathrm{~ms}$ nas 8 tentativas finais do treinamento. A finalidade desse procedimento foi facilitar a familiarização do participante com o tempo de apresentação das palavras alvo. O tempo total de testagem, incluindo as sessões de treinamento e experimental, foi de, aproximadamente, cinco minutos por participante.

\section{Resultados}

Devido a um erro de digitação a palavra estímulo 'ÁSIA' ficou sem o acento agudo ('ASIA') e, portanto, foi excluída das análises estatísticas realizadas. Nesse experimento, a variável dependente é a porcentagem de erros cometidos e, consequentemente, não foi efetuado o cômputo do tempo de reação por condição experimental. No entanto, foi possível o cômputo da média geral do tempo de reação dos participantes que foi de $1007,16 \mathrm{~ms}$, com um desvio padrão de $222,38 \mathrm{~ms}$. No que diz respeito à porcentagem de erros, os participantes apresentaram uma média geral de porcentagem de erros de $32,47 \%$, com um desvio padrão de $26,76 \%$, sendo essa uma distribuição que pode ser considerada normal (Skewness $=0,99$; Kurtosis $=0,50$; Kolmogorov-Smirnov $Z=0,838, p>$ $0,48)$. A Tabela 1 apresenta as estatísticas descritivas da porcentagem de erros por condição experimental para uma manipulação fatorial 2 × 2 de N e NF.

\section{Tabela 1}

Média e Desvio-padrão da Porcentagem de Erros na Tarefa de Identificação Perceptual

\begin{tabular}{lcccc}
\hline & \multicolumn{2}{c}{ N1 } & \multicolumn{2}{c}{ N4+ } \\
\cline { 2 - 5 } & NF0 & NF1 & NF0 & NF1 \\
\hline Média & 31,25 & 39,06 & 21,88 & 37,50 \\
Desvio-Padrão & 35,36 & 34,16 & 28,93 & 35,04 \\
\hline
\end{tabular}

Nota. $\mathrm{N} 1=$ apenas 1 vizinho ortográfico; N4+ = 4 ou mais vizinhos ortográficos; $\mathrm{NF} 0=$ sem vizinhos ortográficos de maior frequência de ocorrência; NF1 = um vizinho ortográfico de maior frequência de ocorrência.

Uma análise de variância considerando $\mathrm{N}$ e NF como fatores intra-sujeitos (within-subjects) com dois níveis cada e considerando o esquema de contrabalanceamento de listas a partir de dois fatores entre-sujeitos (betweensubjects) foi desenvolvida de acordo com as recomendações de Pollatsek e Well (1995) tendo como variável dependente a porcentagem de erros (vide nota 2 para mais informações sobre o design e análise estatística utilizados no presente estudo). Como os fatores entre sujeitos foram incluídos apenas por permitir acrescentar ao termo de erro do teste estatístico a variação referente à interação entre listas e tratamento, os efeitos desses fatores não foram relatados abaixo para facilitar a visualização dos resultados. Assim sendo, a análise estatística realizada indicou que o efeito principal de $\mathrm{N}$ foi facilitador e estatisticamente significante $[F(1,30)=4,31 ; p=0,047$; magnitude do efeito $r=0,35]$. Isto é, as palavras com muitos vizinhos ortográficos foram reconhecidas de forma mais precisa (gerando uma porcentagem de erros $5,5 \%$ menor) do que palavras com poucos vizinhos ortográficos (porcentagem de erros média de $29,69 \%$ e $35,16 \%$, respectivamente). Além disso, também se observou que o efeito principal de NF foi inibidor e estatisticamente significante $[F(1,30)=11,11 ; p<0,01$; magnitude do efeito $r=0,52]$. Ou seja, os participantes apresentaram, aproximadamente, $12 \%$ a mais de erros nas palavras com vizinhos ortográficos de maior frequência de ocorrência em relação às palavras sem vizinhos ortográficos de maior frequência de ocorrência (porcentagem de erros média de $38,28 \%$ e $26,56 \%$, respectivamente). A análise de variância não indicou uma interação estatisticamente significante entre os fatores N e NF $(p>0,35)$.

\section{Discussão}

Os resultados do presente estudo indicaram um efeito facilitador de $\mathrm{N}$ e um efeito inibidor de NF na porcentagem de erros dos participantes. Uma questão a se levantar a esse respeito, nesse e em outros estudos, é se esses efeitos realmente refletem o papel dessas variáveis no processo de acesso lexical ou se esses são o subproduto de algum tipo de estratégia de adivinhação que possa ter sido utilizada pelos participantes. A forma que o presente estudo utilizou para dificultar o uso de estratégias por parte dos participantes foi o estabelecimento de um limite de tempo de 3 segundos para que esses dissessem em voz alta qual era a palavra apresentada. Nesse sentido, pode-se dizer que esse estudo foi bem sucedido, porque o tempo médio de resposta dos participantes foi de cerca de 1 segundo, o que torna muito pouco provável que esses tenham utilizado alguma estratégia sofisticada de adi- 
vinhação ao responder. No entanto, pode-se argumentar que uma estratégia do tipo "falar a primeira palavra que vier à mente" não é uma estratégia tão sofisticada assim e que essa estratégia poderia ser usada em menos de um segundo. Considerando-se que uma estratégia desse tipo privilegiaria as palavras de alta frequência de ocorrência da língua, pois são as que têm maior probabilidade de estar prontamente disponíveis, pode-se argumentar que essa estratégia criaria artificialmente um efeito de NF. Afinal, as palavras com um vizinho ortográfico mais frequente têm um vizinho que pode estar entre as palavras mais frequentes da língua, já, as palavras sem vizinhos ortográficos mais frequentes, não correriam esse risco.

É necessário demonstrar então que essa estratégia não foi usada nesse estudo, ou, se foi, que o seu uso não interferiu nos resultados. Nesse sentido, é importante considerar que a frequência de ocorrência dos estímulos experimentais foi controlada, sendo todas as palavras de baixa frequência (tendo, em média, 10 ocorrências por milhão de palavras). Assim sendo, se os participantes optassem por "falar a primeira palavra que viesse à mente" eles cometeriam um número de erros grande em todas às condições experimentais e não apenas quando houvesse um vizinho de maior frequência. Além disso, ter um vizinho de maior frequência de ocorrência não é equivalente a ter um vizinho de alta frequência. Por exemplo, considerando-se que uma palavra de alta frequência de ocorrência tem uma frequência de, pelo menos, 100 ocorrências por milhão de palavras, pode-se dizer que apenas sete das 64 palavras desse experimento têm um vizinho de maior frequência de ocorrência que também pode ser considerado de alta frequência. Como, de acordo com as estatísticas de Justi e Justi (2008), existem 529 palavras de quatro a seis letras com frequência maior do que 100 ocorrências por milhão de palavras, se algum dos participantes optasse por usar a estratégia de "dizer a primeira palavra que lhe vier à mente" existiriam 529 palavras para ele "chutar" em quaisquer das condições experimentais, sendo que apenas sete dessas palavras são vizinhas ortográficas das palavras empregadas nesse estudo. Destarte, parece pouco provável que o efeito de NF se deva a uma estratégia que envolva "chutar" palavras de alta frequência, porque a frequência de ocorrência dos vizinhos ortográficos das palavras desse estudo não é alta. Além disso, se o efeito de NF se devesse às poucas palavras desse estudo que têm vizinhos ortográficos de alta frequência, muito provavelmente, seu efeito não seria estatisticamente significativo, já que a lógica de se incluir a variação referente ao efeito dos itens no termo de erro do teste estatístico, em certo sentido, visa lidar com esse problema (vide nota 2 e Clark, 1973).

Por fim, uma vantagem adicional desse estudo em relação a outros realizados com essa mesma tarefa, é que, ao requerer respostas vocais, existe uma chance menor, no presente experimento, de que processos específicos relacionados à grafia das palavras possam ter interferido nos resultados (Goldrick et al., 2004). Um cuidado que não foi tomado em outros estudos (Grainger \& Jacobs, 1996; Grainger \& Segui, 1990; Sears, Lupker et al., 1999; Snodgrass \& Mintzer, 1993). Destarte, pode-se concluir que os efeitos de $\mathrm{N}$ e NF observados nesse estudo refletem, muito provavelmente, o papel dessas variáveis no processo de acesso lexical.

Como boa parte dos estudos realizados com a tarefa de identificação perceptual não utilizou respostas vocais (Grainger \& Jacobs, 1996; Grainger \& Segui, 1990; Sears, Lupker et al., 1999; Snodgrass \& Mintzer, 1993), é interessante comparar os resultados do presente estudo com outro estudo que também tenha requerido respostas vocais dos participantes, como o estudo de Carreiras et al. (1997). Comparando-se os resultados desses dois estudos, pode-se perceber que esses são razoavelmente consistentes, afinal, ambos apresentaram um efeito inibidor de NF. A diferença entre os estudos reside na observação de um efeito nulo de $\mathrm{N}$ no estudo de Carreiras et al. (1997) e na observação de um efeito facilitador de $\mathrm{N}$ no presente estudo. Uma das possíveis explicações para essa diferença pode estar no fato de que na tarefa de identificação perceptual com desmascaramento progressivo utilizada por Carreiras et al. (1997), a palavra alvo ficava mais visível com o passar do tempo e isso pode ter induzido os participantes a retardar um pouco suas respostas. Como o TR era a principal variável do estudo de Carreiras et al. (1997), isso pode ter diminuído a precisão de medida e dificultado a detecção do efeito de $\mathrm{N}$ (que no presente estudo foi mais fraco que o efeito de NF). Uma evidência a esse favor é que os participantes do estudo de Carreiras et al. (1997) foram mais lentos do que os participantes do presente estudo (médias de $1446 \mathrm{~ms}$ e $1007 \mathrm{~ms}$, respectivamente), mesmo a tarefa de identificação perceptual padrão podendo ser considerada mais difícil.

No que diz respeito à compatibilidade entre os resultados desse estudo e as predições dos modelos de reconhecimento visual de palavras ${ }^{6}$, pode-se dizer que o efeito inibidor de NF está de acordo com modelo DRC (Coltheart et al., 2001), porque esse modelo pressupõe a existência de um mecanismo de inibição lateral no léxico ortográfico que faz com que as palavras similares umas às outras se inibam mutuamente. Já o efeito facilitador de $\mathrm{N}$ é mais difícil de ser explicado pelo modelo, porque o esperado seria um efeito nulo ou, até mesmo, um efeito inibidor fraco. No entanto, talvez esse efeito não seja tão difícil assim de ser acomodado pelo modelo, uma vez que a tarefa envolve leitura em voz alta. De acordo com simulações computacionais desenvolvidas por Reynolds e Besner (2002), na leitura em voz alta existem dois tipos de fluxo informacional no modelo DRC que podem, em conjunto, reverter o efeito do mecanismo de inibição la-

\footnotetext{
${ }^{6}$ Por questões de espaço serão discutidos aqui apenas os modelos DRC (Coltheart et al., 2001) e os modelos de Processamento Paralelo Distribuído (Plaut et al., 1996; Seidenberg \& McClelland, 1989), por poderem ser considerados os mais influentes na área.
} 
teral: (a) a reverberação da ativação proveniente do léxico ortográfico ao nível das letras pode facilitar o reconhecimento de letras compartilhadas pela palavra alvo e seus vizinhos ortográficos; $\mathrm{e}$, (b) a ativação repassada pelo léxico ortográfico ao léxico de output fonológico e desse último ao nível de fonema, pré-ativariam fonemas compartilhados pela palavra alvo e seus vizinhos ortográficos. Dessa forma, a explicação para um efeito facilitador de $\mathrm{N}$ dependeria da força das conexões entre o léxico ortográfico e o nível da letra e entre o léxico ortográfico, o léxico de output fonológico e o nível de fonema. Se a força dessas conexões for razoável, elas poderiam reverter o efeito do mecanismo de inibição lateral. Isso não afetaria o efeito de NF, porque um único vizinho ortográfico, mesmo que mais frequente, enviaria pouca ativação para outros níveis do modelo por se tratar de uma única fonte de informação (já palavras com muitos vizinhos ortográficos teriam várias fontes de informação ativando unidades em comum em diferentes níveis do modelo) e, além disso, como a inibição no léxico ortográfico é proporcional ao nível de ativação das unidades, um vizinho ortográfico mais frequente torna-se ativo mais rapidamente tornando-se um inibidor mais forte. Se essa hipótese estiver correta, é de se esperar que o efeito de $\mathrm{N}$, mas não o de NF, seja modulado também pelo tipo de resposta envolvida na tarefa, porque tarefas que impliquem em uma demanda mais forte a processos fonológicos (como gerar os fonemas necessários à pronúncia de um estímulo) se beneficiariam mais da ativação repassada pelo léxico de output fonológico ao nível de fonema e esse processo é mais sensível a $\mathrm{N}$ do que a NF. Uma evidência de que esse, de fato, pode ser o caso é que, nos estudos realizados por Justi e Pinheiro $(2006,2008)$ com falantes do português brasileiro, $\mathrm{N}$ apresentou um efeito inibidor na tarefa de decisão lexical do tipo Go/NoGo que é uma tarefa que não envolve respostas vocais.

Considerando-se os modelos de Processamento Paralelo Distribuído (PPD) de Plaut et al. (1996) e de Seidenberg e McClelland (1989), no que diz respeito às tarefas que envolvem gerar a pronúncia de uma palavra, pode-se dizer que os efeitos de N e NF são facilitadores nesses modelos (conforme demonstrado nas simulações computacionais desenvolvidas por Sears, Hino, \& Lupker, 1999). Os efeitos seriam facilitadores porque as unidades responsáveis por gerar a pronúncia de uma palavra alvo, durante o processo de treinamento, beneficiar-seiam também do treino com as palavras que fossem ortograficamente similares à palavra alvo. Assim, durante o treinamento, palavras com muitos vizinhos ortográficos ou com vizinhos ortográficos mais frequentes fortaleceriam as conexões responsáveis por gerar a pronúncia dos grafemas compartilhados nessas palavras, e, durante a pronúncia, as conexões mais fortalecidas resultariam em mais precisão e rapidez no processamento das palavras que as compartilham (Seidenberg \& McClelland, 1989). Assim sendo, se considerarmos os dados desse estudo, os modelos PPD seriam compatíveis com o efeito facilitador de $\mathrm{N}$, mas teriam dificuldades em acomodar o efeito inibidor de NF. No entanto, nos modelos PPD, acomodar os diferentes efeitos de N e NF parece implicar em dificuldades mais centrais do que as do modelo DRC (Coltheart et al., 2001). Isso ocorre porque nos modelos PPD, os efeitos de N e NF são derivados de um mesmo mecanismo: o benefício que as unidades responsáveis por gerar o padrão de ativação de uma palavra alvo obtêm do treino com palavras similares a essa palavra alvo. Desse modo, em princípio, os efeitos de N e NF, nos modelos PPD, devem sempre apresentar a mesma direção facilitadora, sendo difícil para esses modelos acomodar, ao mesmo tempo, um efeito facilitador de $\mathrm{N} \mathrm{e}$ um efeito inibidor de NF.

\section{Conclusão}

$\mathrm{O}$ presente estudo traz evidências de que $\mathrm{N}$ tem um efeito facilitador e de que NF tem um efeito inibidor no processo de acesso lexical. É pouco provável que esses resultados se devam a estratégias sofisticadas de adivinhação porque o tempo médio de resposta dos participantes foi de apenas um segundo. Assim sendo, esses resultados trazem dificuldades para modelos atuais de reconhecimento visual de palavras porque esses tendem a prever ou efeitos inibidores de ambas as variáveis (modelo DRC - Coltheart et al., 2001), ou efeitos facilitadores de ambas as variáveis (modelos PPD - Plaut et al., 1996; Seidenberg \& McClelland, 1989). Destarte, é importante que trabalhos futuros investiguem se modificações nos parâmetros desses modelos seriam capazes de simular efeitos facilitadores de $\mathrm{N}$ e efeitos inibidores de NF ao mesmo tempo.

\section{Referências}

Andrews, S. (1989). Frequency and neighborhood effects on lexical access: Activation or search? Journal of Experimental Psychology: Learning, Memory \& Cognition, 1, 802-814. Andrews, S. (1997). The effect of orthographic similarity on lexical retrieval: Resolving neighborhood conflicts. Psychonomic Bulletin \& Review, 4, 439-461.

Arduino, L., \& Burani, C. (2004). Neighborhood effects on nonword visual processing in a language with shallow orthography. Journal of Psycholinguistic Research, 33, 7595.

Carreiras, M., Perea, M., \& Grainger, J. (1997). Effects of orthographic neighborhood in visual word recognition: Crosstask comparisons. Journal of Experimental Psychology: Learning, Memory, \& Cognition, 23, 857-871.

Clark, H. (1973). The language-as-fixed-effect fallacy: A critique of language statistics in psychological research. Journal of Verbal Learning and Verbal Behavior, 12, 335-359.

Coltheart, M., Davelaar, E., Jonasson, J., \& Besner, D. (1977). Access to the internal lexicon. In S. Dornic (Ed.), Attention and performance (Vol. 6, pp. 535-555). Hillsdale, NJ: Erlbaum. 
Justi, F. R. R. \& Roazzi, A. (2012). Efeitos de Vizinhança Ortográfica no Português Brasileiro: Um Estudo com a Tarefa de Identificação Perceptual.

Coltheart, M., Rastle, K., Perry, C., Langdon, R., \& Ziegler, J. (2001). DRC: A dual route cascaded model of visual word recognition and reading aloud. Psychological Review, 108, 204-256.

Forster, K., \& Forster, J. (2003). DMDX: A windows display program with millisecond accuracy. Behavior Research Methods, Instruments and Computers, 35, 116-124.

Goldrick, M., Folk, J. R., \& Rapp, B. (2004). Orthographic neighborhoods: Evidence from dysgraphia. Brain and Language, 91, 94-95.

Grainger, J., \& Jacobs, A. (1996). Orthographic processing in visual word recognition: A multiple read-out model. Psychological Review, 103, 518-565.

Grainger, J., O’Reagan, K., Jacobs, A., \& Segui, J. (1989). On the role of competing word units in visual word recognition: The neighborhood frequency effect. Perception \& Psychophysics, 45, 189-195.

Grainger, J., \& Segui, J. (1990). Neighborhood frequency effects in visual word recognition: A comparison of lexical decision and masked identification latencies. Perception \& Psychophysics, 47, 191-198.

Huntsman, L., \& Lima, S. (1996). Orthographic neighborhood structure and lexical access. Journal of Psycholinguistic Research, 25, 417-429.

Huntsman, L., \& Lima, S. (2002). Orthographic neighbors and visual word recognition. Journal of Psycholinguistic Research, 31, 289-306.

Justi, F. R. R., \& Justi, C. N. G. (2008). As estatísticas de vizinhança ortográfica do português e do inglês são diferentes? Psicologia em Pesquisa (Juiz de Fora), 2, 61-73.

Justi, F. R. R., \& Pinheiro, A. M. V. (2006). O efeito de vizinhança ortográfica no português do Brasil: Acesso lexical ou processamento estratégico. Interamerican Journal of Psychology, 40, 275-288.

Justi, F. R. R., \& Pinheiro, A. M. V. (2008). O efeito de vizinhança ortográfica em crianças brasileiras: Estudo com a tarefa de decisão lexical. Interamerican Journal of Psychology, 42, 559-569.

Mathey, S. (2001). L'influence du voisinage orthographique lors de la reconnaissance des mots écrits. Revue Canadienne de Psychologie Expérimentale, 55, 1-23.

Mathey, S., \& Zagar, D. (2006). The orthographic neighbourhood frequency effect in French: A letter-case manipulation study. Canadian Journal of Experimental Psychology, 60 159-165.

Monsell, S., Doyle, M., \& Haggard, P. (1989). Effects of frequency on word recognition tasks: Where are they? Journal of Experimental Psychology: General, 118(1), 43-71.

Núcleo Interinstitucional de Lingüística Computacional. (2005). Corpus NILC / São Carlos v.7.1. Retrieved August 30, 2005, from http://www.nilc.icms.usp.br/nilc/tools/corpora.htm

Perea, M., Carreiras, M., \& Grainger, J. (2004). Blocking by word frequency and neighborhood density in visual word recognition: A task-specific response criteria account. Memory \& Cognition, 32, 1090-1102.

Perea, M., \& Pollatsek, A. (1998). The effects of neighborhood frequency in reading and lexical decision. Journal of Experimental Psychology: Human Perception \& Performance, 24,767-779.

Perea, M., \& Rosa, E. (2000). The effects of orthographic neighborhood in reading and laboratory word identification tasks: A review. Psicológica, 21, 327-340.
Pinheiro, A. M. V., \& Reis, C. (2004). A influência da natureza fonética do fonema inicial da palavra na medida de tempo de reação em tarefas de leitura em voz alta. Revista de Estudos da Linguagem, 12, 19-36.

Plaut, D., McClelland, J., Seidenberg, M., \& Patterson, K. (1996). Understanding normal and impaired word reading: Computational principles in quasi-regular domains. Psychological Review, 103, 56-115.

Pollatsek, A., Perea, M., \& Binder, K. (1999). The effects of neighborhood size in reading and lexical decision. Journal of Experimental Psychology: Human Perception \& Performance, 25, 1142-1158.

Pollatsek, A., \& Well, A. D. (1995). On the use of counterbalanced designs in cognitive research: A suggestion for a better and more powerful analysis. Journal of Experimental Psychology: Learning, Memory, and Cognition, 21, 785-794.

Raaijmakers, J. (2003). A further look at the "language-as-fixedeffect fallacy". Canadian Journal of Experimental Psychology, 57, 141-151.

Raaijmakers, J., Schrijnemakers, J., \& Gremmen, F. (1999). How to deal with "the language-as-fixed-effect fallacy": Common misconceptions and alternative solutions. Journal of Memory and Language, 41, 416-426.

Reynolds, M., \& Besner, D. (2002). Neighbourhood density effects in reading aloud: New insights from simulations with the DRC Model. Canadian Journal of Experimental Psychology, 56, 310-318.

Rosenthal, R. (1984). Meta-analytic procedures for social research. Beverly Hills, CA: Sage.

Sears, C., Campbell, C., \& Lupker, S. (2006). Is there a neighborhood frequency effect in English? Evidence from reading and lexical decision. Journal of Experimental Psychology: Human Perception and Performance, 32, 10401062.

Sears, C., Hino, Y., \& Lupker, S. (1999). Orthographic neighborhood effects in parallel distributed processing models. Canadian Journal of Experimental Psychology, 53, 220-229.

Sears, C., Lupker, S., \& Hino, Y. (1999). Orthographic neighborhood effects in perceptual identification and semantic categorization tasks: A test of the multiple read-out model. Perception and Psychophysics, 61, 1537-1554.

Seidenberg, M., \& McClelland, J. (1989). A distributed developmental model of word recognition and naming. Psychological Review, 96, 523-568.

Siakaluk, P., Sears, C., \& Lupker, S. (2002). Orthographic neighborhood effects in lexical decision: The effects of nonword orthographic neighborhood size. Journal of Experimental Psychology: Human Perception and Performance, 28, 661-681.

Snodgrass, J., \& Minzer, M. (1993). Neighbourhood effects in visual word recognition: Facilitatory or inhibitory? Memory \& Cognition, 21, 247-266. 


\section{Anexo A}

\section{Estímulos Utilizados na Tarefa de Identificação Perceptual}

Palavras correspondentes à manipulação fatorial de $\mathrm{N}$ e NF:

$$
\mathrm{N}=1 \text { vizinho ortográfico }
$$

$$
\mathrm{NF}=0 \text { vizinhos }
$$

Lista 1

ASTRO
BISPO
DUPLAS
FAUNA

Lista 2

BICHOS

CRENÇA

SAQUE

TRUQUE

Lista 3

CAPIM

FILTRO

MANHÃS

PADRES

Lista 4

$\begin{array}{ll}\text { CACAU } & \text { CARNÊS } \\ \text { CREDOR } & \text { CORAIS } \\ \text { FIBRAS } & \text { FRANGO } \\ \text { TAMPÃO } & \text { TRONO }\end{array}$

$$
\mathrm{NF}=1 \text { vizinho }
$$

ONZE *

BALCÃO

DIVÃ

FICHAS

BONÉ

CRISES

SABOR

TRIBO

CRIVO

FUSCA

MUNDOS

PATRÃO

CARNÊS

TRONO
$\mathrm{N}>4$ vizinhos ortográficos

\begin{tabular}{cc}
\multicolumn{2}{c}{$\mathrm{N}>4$ vizinhos ortográficos } \\
$\mathrm{NF}=0$ vizinhos & $\mathrm{NF}=1$ vizinho \\
ASAS & ÁSIA \\
BURRO & BARES \\
DICAS & DATAS \\
FERAS & FADAS \\
BRIGAS & BARRAS \\
CALMA & CABOS \\
SUCO & SACO \\
TAMPA & TRAVE \\
CRAVO & CABRA \\
FARDOS & FUNDA \\
MAPAS & MUROS \\
PINGA & PICOS \\
CASCA & CALDO \\
CONDE & CALÇA \\
FITAS & FUMO \\
TUMBA & TOMBO
\end{tabular}

* O fonema inicial /õ/ foi pareado com o fonema inicial /a/ das palavras 'ASTRO', 'ASAS' e 'ÁSIA', porque Pinheiro e Reis (2004) não observaram diferenças estatisticamente significativas entre as médias de tempo de reação das palavras iniciadas com esses fonemas (/õ/ e /a/). 